\title{
Self-Assembled Orientation of Polymer Chains in Methylcellulose Gel during Drying Process
}

\author{
By Reiko NISHIDA* and Masae TAKAHASHI
}

By the study of drying process of methylcellulose aqueous solution, the behavior of the polymer chains in physical gel became clear.

Methylcellulose powder was dissolved in water and kept at an adequate condition to be in physical gel state during the drying process. Morphological time change of a cap-shaped gel droplet on a glass plate was observed by polarized optical microscope (POM) and wide angle X-ray scattering (WAXS) apparatus during and after the drying process until the droplet was completely dried up to be a solidified thin film. By POM observation, the whole image of the thin film showed just as a single negative spherulite crystallite, and the film measured by WAXS showed that its ac plain is parallel to the substrate plane on the assumption that the methylcellulose crystal structure is same as trimethylcellulose.

This suggests that polymer chains in the methylcellulose gel rearranged nearly on concentric circles around the center of the sample. This alignment suggests that the motion of polymer chains is occurred along the periphery of the gel droplet in the drying process and a self-assembled orientation of the polymer chains limited by the periphery is induced to reduce the total free energy in the solidified thin film.

KEY WORDS: Self-Assembled Orientation / Drying Process / Methylcellulose / Physical Gel / Polarized Optical Microscope / Wide

Angle X-ray Scattering /

Drying phenomena of gels have been interested in by many workers. ${ }^{1-5}$ Most of the works have been related to the chemical gels in which the molecules at a cross-link are tightly bound each other by covalent bond. Few reports, however, have been reported on the dried physical gels in which the molecules at the cross-links are rather loosely bound by weak interactions such as van der Waals interaction or hydrogen bond.

We have been interested in the behavior of polymer chains in the physical gels with various condition of concentrations and temperatures. ${ }^{6,7}$ In particular, we have studied the drying process of methylcellulose (MC) aqueous solution which is one of the most typical physical gels and shows a thermally reversible sol-gel transition.

The MC solution was found to form solid thin films which have a characteristic MC molecules orientation by keeping the gel state using by phase diagrams of our previous work. ${ }^{6}$ The cross-links of molecular chains in physical gels are expected to move and to rearrange with the progress of the evaporation to reduce the total free energy. This motion of molecular chains would be limited by the boundary of the sample and cause a characteristic morphology by a self-assembled reaction.

We consider that the self-assembled reaction in the MC solution affects either the formation of the hydrophilic or hydrophobic surface of polymer films. MC molecules have structural anisotropy for having hydrophilic and hydrophobic part in the cell unit ${ }^{8}$ and thus the surface wettability will be determined by the self-assembled orientation of the part.

The purpose of the present study is to make a detailed observation on the drying process of the MC gel by polarized optical microscope (POM) and to elucidate the distribution and arrangement of polymer chain molecules in the dried MC gel which are affected by the interaction among the molecules in the gel state thin films by POM and wide angle X-ray scattering (WAXS) apparatus.

\section{EXPERIMENTAL}

\section{Sample Preparation}

Molecular structure of MC is shown in Figure 1. The degree of substitution (DS) of the methyl group could be controlled from 0 to 3. Since MC with $\mathrm{DS}=1.6-2.0$ dissolves in cold water, we prepared aqueous solutions with the 4 kinds of MC powders whose DSs are fixed between 1.75 and 1.79 and molecular weights ranged from $1.24 \times 10^{5}$ to $9.36 \times 10^{5}$ as shown in Table I. Their properties are summarized in the literature $^{6}$ and the details of the method of preparation of MC aqueous solutions are also reported in it.

As an example showing sol-gel transition in the sample, the phase diagram of the MC-4 sample for the temperature vs. concentration relation determined in our previous report ${ }^{6}$ is repeated in Figure 2 in a simplified manner. It was recognized that the MC solution was found in some drying conditions, to form solid thin films keeping the gel state. In the present study, the initial concentration $C_{0}$ is determined to satisfy a sol condition at room temperature, typically $1 \mathrm{wt} \%$ and the drying temperature $T_{0}$ is fixed to keep the sample at gel state, typically $45^{\circ} \mathrm{C}$. 

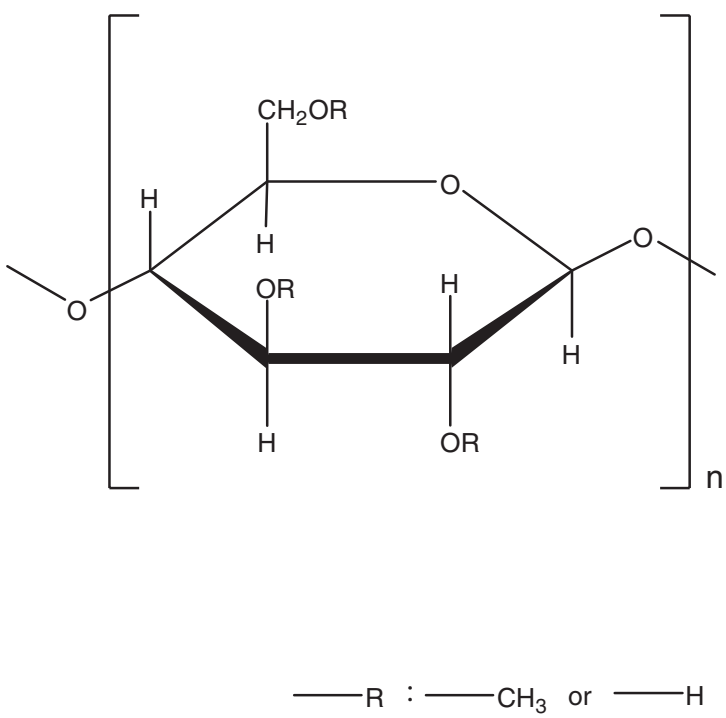

Figure 1. Molecular structure of methylcellulose. The DS is defined by the number of $\mathrm{CH}_{3}$ groups in position $\mathrm{R}$ relative to the total number of $\mathrm{R}$

Table I. Molecular characteristics of methylcelluloses

\begin{tabular}{cccc}
\hline Samples & $\begin{array}{c}\text { Degree of } \\
\text { Substitution }\end{array}$ & $\begin{array}{c}\text { Weight-Average } \\
\text { Molecular Weight }\end{array}$ & $\begin{array}{c}\text { Degree of } \\
\text { Polymerization }\end{array}$ \\
\hline MC-1 & 1.77 & $1.24 \times 10^{5}$ & $0.64 \times 10^{3}$ \\
\hline MC-2 & 1.75 & $3.57 \times 10^{5}$ & $1.91 \times 10^{3}$ \\
\hline MC-3 & 1.79 & $7.83 \times 10^{5}$ & $4.19 \times 10^{3}$ \\
\hline MC-4 & 1.78 & $9.36 \times 10^{5}$ & $5.01 \times 10^{3}$ \\
\hline
\end{tabular}

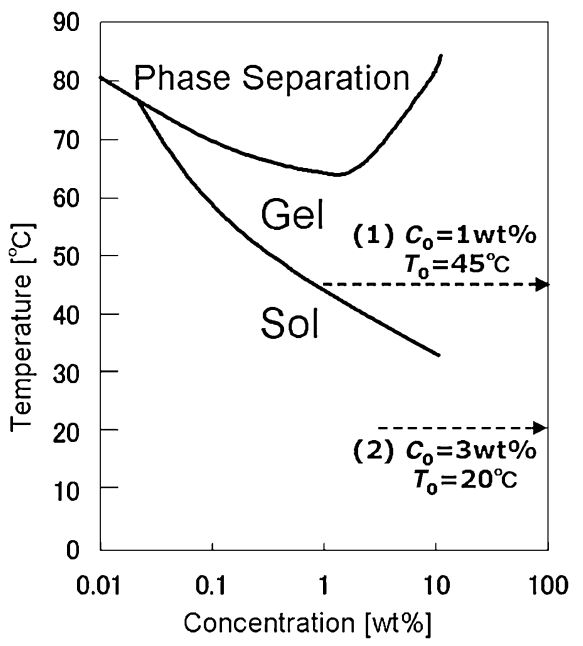

Figure 2. Simplified experimental phase diagram for methylcellulose (MC-4) aqueous solution (for details, see Reference 6). Two horizontal dashed lines represent the trajectories of drying process: (1) kept at gel state $C_{0}=1 \mathrm{wt} \%, T_{0}=45^{\circ} \mathrm{C}$ and (2) kept at sol state $C_{0}=$ $3 \mathrm{wt} \%, T_{0}=20^{\circ} \mathrm{C}$.

A small amount of the MC aqueous solution ( $c a$. 10-100 $\mathrm{mg}$ ) is dropped onto a surface of a glass plate using a syringe to form a cap-shaped droplet with a diameter of $c a .5 \mathrm{~mm}-10 \mathrm{~mm}$. (a)

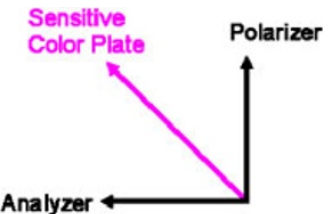

(b)

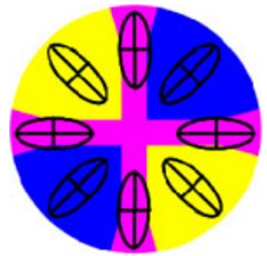

(c)

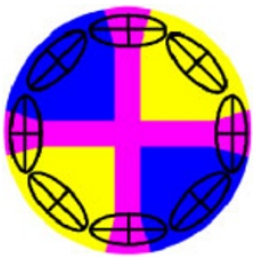

\section{$\bigoplus$ : Optical Uniaxial Crystal}

Figure 3. Schematic diagram by adding sensitive color plate in POM (a) inserted direction of sensitive color plate toward polarizer and analyzer. Observation images of (b) positive spherulite and (c) negative spherulite. Ellipses in (b) and (c) are symbolized optical uniaxial crystal.

The glass plate was put on a hot stage (Linkam LK-600PM) to dry up the droplet at the constant temperature $T_{0}$.

Furthermore, in order to investigate the effect of the shape of the periphery on the structure and the arrangement motion of polymer chains, a small rectangular glass plate was prepared and its whole surface was covered by the solution. Then, the rectangular sample was dried up to investigate influence of the boundary shape.

\section{POM Observation and X-ray Diffraction}

The structure change of the MC gel from the droplet to the solidified thin film is successively observed by taking photographs using a polarized optical microscope (POM: Nikon OPTIPHOT2-POL) by using crossed-nicol. Furthermore, to obtain a direction of molecular orientation, a sensitive color plate was inserted with rotating $-\pi / 4$ around the polarizer direction to provide a retardation of $530 \mathrm{~nm}$ as shown in Figure 3a. With the plate, schematic images of a positive spherulite and a negative spherulite are drawn with blue and yellow color patterns caused by a direction of ordinary and extraordinary rays of birefringence as shown in Figure $3 b$ and $3 \mathrm{c}$.

The crystalline morphology of the solidified thin films without removing from the glass substrates were investigated by wide angle X-ray scattering apparatus (WAXS: Rigaku RINT-1500; target: $\mathrm{Cu}-\mathrm{K}_{\alpha} ; 40 \mathrm{kV}, 200 \mathrm{~mA}$ ) with reflection method for the surface of the film between $2 \theta=5^{\circ}$ to $40^{\circ}$ with a step size $2 \theta=0.02^{\circ}$. 

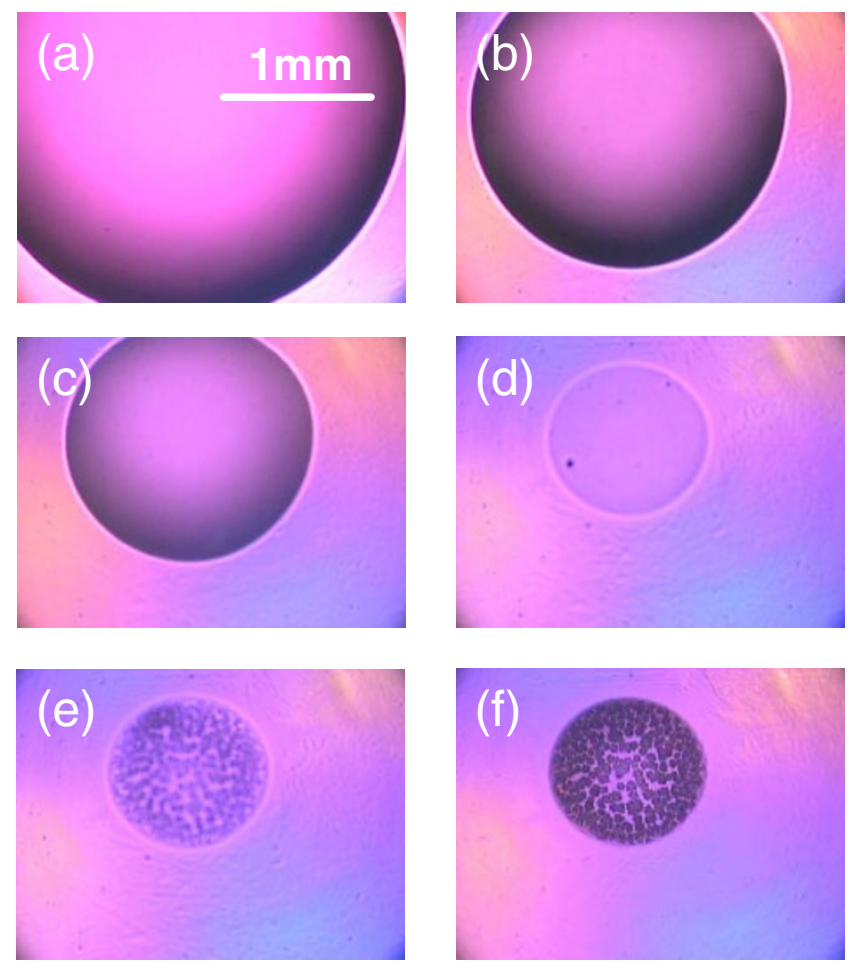

Figure 4. A typical result of drying process from a MC gel droplet (MC-4, $C_{0}=1 \mathrm{wt} \%, T_{0}=45^{\circ} \mathrm{C}$ ) to a solidified thin film observed by POM inserted sensitive color plate, and these images are enlarged at the center part of the droplet. Each elapsed time from start of drying process: (a) $860 \mathrm{~s}$, (b) $960 \mathrm{~s}$, (c) $1005 \mathrm{~s}$, (d) $1058 \mathrm{~s}$, (e) $1060 \mathrm{~s}$, (f) $1061 \mathrm{~s}$. (a), (b), (c): The dried regions had increased and the swollen region had decreased from the periphery toward the center of the droplet, (d): completely dried up and (e), (f): a small circular conformation appeared in a short time at the center of the film.

\section{RESULTS}

Typical Structure of a Thin Film Obtained from Drying Process of a MC Gel Droplet

A POM observation of drying process of a MC gel droplet at the condition of $C_{0}=1 \mathrm{wt} \%$ and $T_{0}=45^{\circ} \mathrm{C}$ was carried out using a sensitive color plate. The photographs of the center part of the droplet taking at several time intervals are shown in Figure $4 \mathrm{a}-4 \mathrm{f}$. Dried region appears at the periphery of the droplet and it proceeds toward the center, while the swollen region decreases keeping the cap shape during the drying process as shown in Figure $4 \mathrm{a}-4 \mathrm{c}$.

The process seems to be completed to form a thin film after about $1000 \mathrm{~s}$ as shown in Figure 4d. A smaller concentric circular conformation with $c a .1 \mathrm{~mm}$ diameter appeared after the dried up at the center part as shown in Figure $4 \mathrm{e}$ and $4 \mathrm{f}$. This part was assured to be consisted with a number of dendrite crystals by X-ray diffraction. The detailed investigation of this part will be appeared elsewhere.

In order to understand the total features of the sample, whole image of the sample is shown in Figure 5. A single large Maltese cross pattern was observed by POM with crossed-nicol as shown in Figure 5a, and blue and yellow color patterns (a)

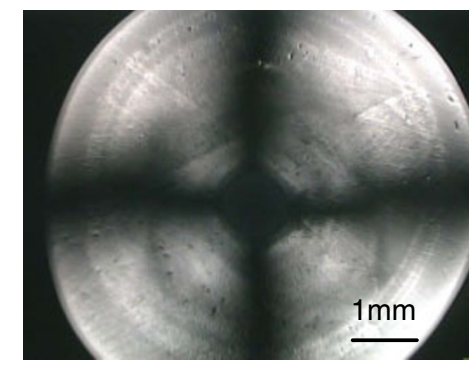

(b)

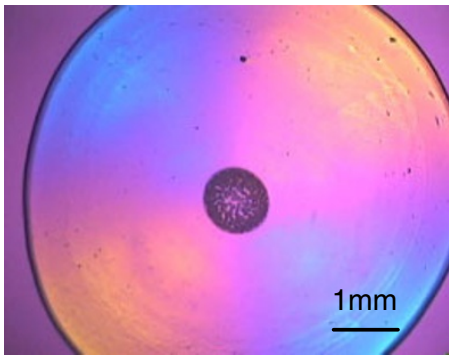

Figure 5. Whole image of the dried thin film of $\mathrm{MC}$ gel (the same film of Figure 4) observed by POM: (a) with crossed-nicol and (b) added sensitive color plate.

appeared for adding a sensitive color plate as shown in Figure $5 \mathrm{~b}$. The color patterns are cause by the birefringence of MC molecule. The whole of the thin film consists of just as a single negative spherulite crystallite (in Figure $3 \mathrm{c}$ ). The area of the thin film region was almost the same as the contact area of the droplet with the glass substrate at the beginning of the drying process.

\section{Dried from MC Sol Solution}

As has been described above, dried film from MC gel produced a single negative spherulite-like pattern. However, it has been reported ${ }^{9}$ that not only negative spherulite crystals, but a number of positive spherulite crystals with higher order crystal structures have been formed from melted or supersaturated polymer solutions. In order to investigate the effect of the starting condition of MC solution, we prepared a MC sol solution and its drying process was carried out.

The MC sol solution was prepared according to the phase diagram shown in Figure 2. The drying process was performed at the condition of $C_{0}=3 \mathrm{wt} \%$ and $T_{0}=20^{\circ} \mathrm{C}$. The observed image of the dried thin film was shown in Figure 6. Many positive spherulite crystals have been observed and no dendrite crystals appeared in the center of the thin films produced from gel as shown in Figure 4. Hence, the single negative spherulitelike pattern and dendrite crystals seem to be very peculiar to the dried film from MC gel.

Next, we consider the reason for showing the single negative spherulite-like pattern at the dried thin film from MC gel droplet. In general, polymer crystals including MC have an optical uniaxial anisotropy and the direction of the polymer chain is equivalent to the long axis of the optical uniaxial crystal. Thus, in the MC thin films kept to be in gel state during 
(a)

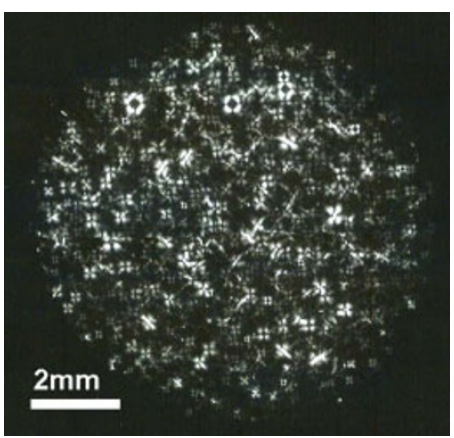

(b)

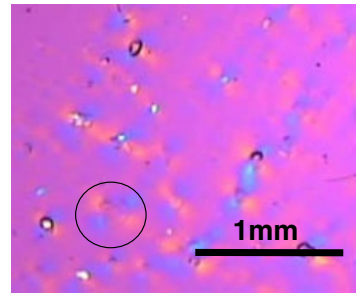

Figure 6. Images of the dried thin film of MC sol (MC-4, $C_{0}=3 \mathrm{wt} \%, T_{0}=$ $20^{\circ} \mathrm{C}$ ) observed by POM: (a) whole image with crossed-nicol and (b) enlarged image added sensitive color plate. The film is composed of a number of positive spherulites (emphasized one positive spherulite pattern by drawn a circle in (b)).

drying process, the polymer chains of MC are perpendicular to the radial axis of the dried circular film and so, polymer chains of MC are arranged on concentric circles.

\section{Effect of Initial Concentration, Drying Temperature and Molecular Weight}

Dependences of the dried MC gel thin film structures on initial concentration, drying temperature and molecular weight were investigated. Results of MC- 1 and MC- 4 solutions are shown as a characteristic example, because the molecular weight of MC-1 is smallest in our MC samples shown in Table I, and the molecular weight of MC- 1 is about $1 / 8$ of MC-4 which is used as standard MC powder in the present study. According to our experimental phase diagram of MC- $1{ }^{6}$ the sol-gel transition temperature is about $55^{\circ} \mathrm{C}$ at $1 \mathrm{wt} \%$, $45^{\circ} \mathrm{C}$ at $2 \mathrm{wt} \%$ and $35^{\circ} \mathrm{C}$ at $10 \mathrm{wt} \%$.

To investigate the molecular weight effects, we compared the thin films by $C_{0}=2 \mathrm{wt} \%$ of MC- 1 solution and $C_{0}=$ $1 \mathrm{wt} \%$ of MC-4 solution at same temperature $T_{0}=45^{\circ} \mathrm{C}$ which were dried keeping gel state. As shown in Figures 5 and 7a, more deeply colored and more homogeneous POM patterns are obtained for larger molecular weight MC gels. In comparison with initial concentration of $C_{0}=2 \mathrm{wt} \%$ (Figure 7a) and $C_{0}=10 \mathrm{wt} \%$ (Figure 7b) of MC-1 solution at $T_{0}=45^{\circ} \mathrm{C}$, more deeply colored and more homogeneous POM patterns are obtained for higher initial concentration of MC gels. But the difference of the drying temperature did not much affect on the homogeneity of POM patterns, as shown in Figure $7 \mathrm{~b}\left(T_{0}=45^{\circ} \mathrm{C}\right)$ and Figure $7 \mathrm{c}\left(T_{0}=35^{\circ} \mathrm{C}\right)$.

We conclude that all the thin films formed by the dried MC gel shows a similar pattern to those of a negative spherulite (a)

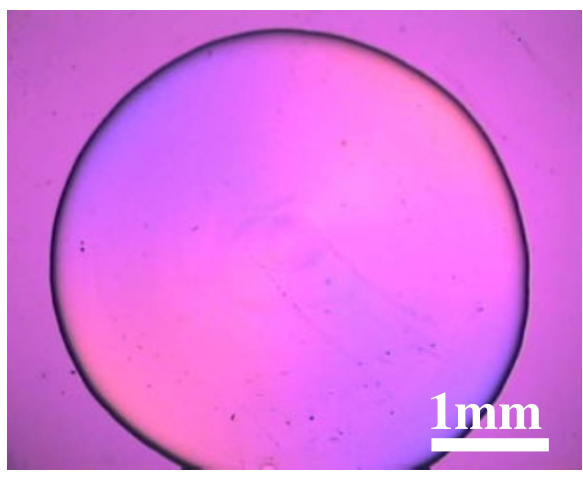

(b)

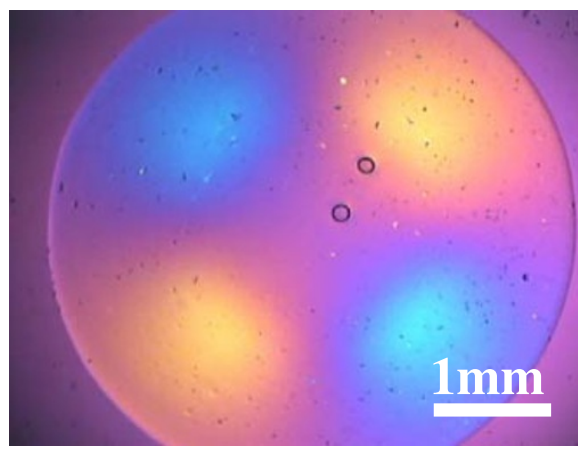

(c)

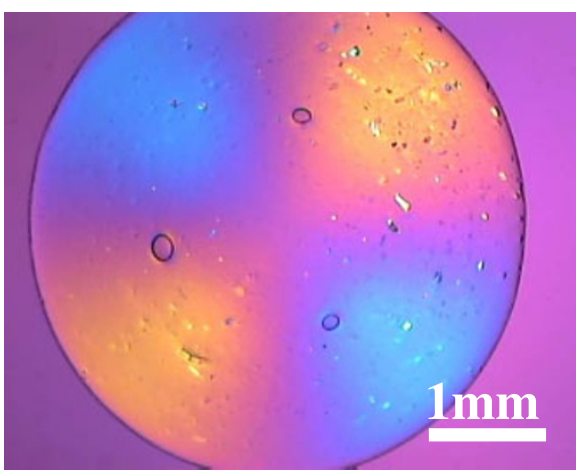

Figure 7. Images of the dried films from MC gels observed by POM inserted sensitive color plate. They were made from the MC powder of lower molecular weight (MC-1): (a) low initial concentration $C_{0}=$ $2 \mathrm{wt} \%, T_{0}=45^{\circ} \mathrm{C}$, (b) high initial concentration $C_{0}=10 \mathrm{wt} \%$, $T_{0}=45^{\circ} \mathrm{C}$ and (c) $C_{0}=10 \mathrm{wt} \%, T_{0}=35^{\circ} \mathrm{C}$ which is lowest temperature to be gel state at $C_{0}=10 \mathrm{wt} \%$.

regardless of initial concentration, drying temperature and molecular weight. It seems that the appearance of the single negative spherulite-like pattern is peculiar to the drying process of gels.

The dendrite structure shown in Figure 5 at the center part of the dried thin film disappeared with decreasing molecular weight of MC. Furthermore, from various films dried up by another condition, we consider that the dendrite structures appear at optimal condition of initial concentration, drying temperature and molecular weight. 


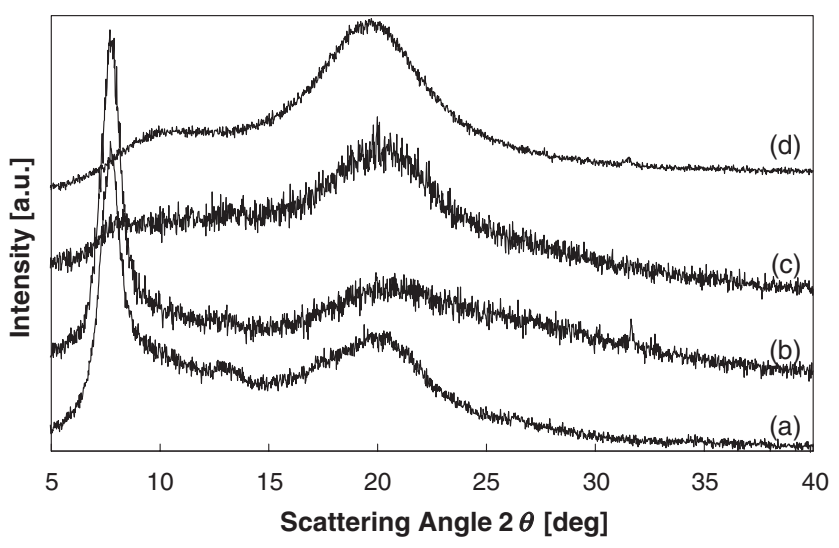

Figure 8. X-Ray diffraction patterns measured by WAXS: (a) dried film keeping gel state (MC-1, $C_{0}=10 \mathrm{wt} \%, T_{0}=40^{\circ} \mathrm{C}$ ), (b) dried film keeping gel state (MC-4, $\left.C_{0}=1 \mathrm{wt} \%, T_{0}=60^{\circ} \mathrm{C}\right)$, (c) dried film keeping sol state $\left(\mathrm{MC}-4, C_{0}=3 \mathrm{wt} \%, T_{0}=10^{\circ} \mathrm{C}\right.$ ) and (d) $\mathrm{MC}-4$ powder which is used as a solute.

\section{Crystalline Morphology of dried MC Thin Films}

The crystalline morphology of the dried MC thin film was investigated by WAXS. As shown in Figure $8 \mathrm{a}$ and $8 \mathrm{~b}$, clear diffraction peaks at around $2 \theta=8^{\circ}$ were observed for a dense, homogeneous colored, negative spherulite samples, where the initial concentration $C_{0}=1 \mathrm{wt} \%$ of MC- 4 solution with the drying temperature $T_{0}=60^{\circ} \mathrm{C}$ and the initial concentration $C_{0}=10 \mathrm{wt} \%$ of $\mathrm{MC}-1$ solution with the drying temperature $T_{0}=40^{\circ} \mathrm{C}$. Comparing the two profiles, we conclude that the existence of dendrite crystals does not affect the diffraction peak at around $2 \theta=8^{\circ}$ because MC- 1 film does not have dendrite crystal. The diffraction patterns from a MC powder sample and from a thin film dried at a sol state are also shown in Figure 8 for the comparison and they showed almost diffuse patterns and are regarded as amorphous structure. Therefore, we note that the thin film dried at a gel state has a particular planer orientation.

We assume that the crystal lattice of MC is same as trimethylcellulose (TMC) because the substitutions of MC are all replaced by methyl groups in TMC. Furthermore, Kato et al. have reported that the peak position of X-ray diffraction pattern of cast films from MC aqueous solution well coincided with TMC. ${ }^{10}$ The crystal structure of TMC is regarded as orthorhombic with four chains running through the unit cell and the values of axes are $a=0.46 \mathrm{~nm}, \mathrm{~b}=4.32 \mathrm{~nm}$ and $\mathrm{c}=1.04 \mathrm{~nm}$ (fiber axis), which have been analyzed by Zugenmaier et al. by using X-ray and electron diffraction data. ${ }^{11}$

The diffraction peak at $2 \theta=7.7^{\circ}$ of the gel originated solidified thin film means that a spacing between the diffracting planes is about $1.15 \mathrm{~nm}$ and its miller index are considered as either (001) or (040) but the peak of (001) corresponds to the molecular chains standing perpendicularly on the substrate plane. This is not consistent with the POM images. The POM images indicate the polymer chains lay on the substrate plane in the thin film and the peak of (040) corresponds to the ac plane in parallel with the substrate plane. Hence, we suppose that (040) is suitable in our experiment.

\section{DISCUSSION}

\section{Homogeneity of Negative Spherulite Pattern}

The degree of the homogeneity of the POM color of the dried MC thin film depends on the drying condition. As have been mentioned, MC initial concentration and molecular weight of MC are related to the homogeneity of the color. The observation proved that the higher concentration and larger molecular weight cause the better homogeneity.

The homogeneity is considered to depend on the mobility of the MC molecules. The higher concentration increases the entanglement of the polymer chains and their movement is hindered. This effect of hindrance is probably enhanced for the polymer molecules of larger molecular weight. The higher concentration and larger molecular weight contribute to the reduction of the mobility of the $\mathrm{MC}$ molecules and cause to keep the homogeneity of the MC solution.

\section{Formation Process of the Spherulite during the Drying Process}

Our experiments have shown that the spherulite-like pattern was observed by POM kept to be in gel state during drying process. The spherulite crystal is usually generated in the nucleation and growth process from the condensed or melted solution of polymers. Our results suggest the similar process has occurred.

The dried region appeared at the periphery of the cap-shaped MC gel. The molecular density in the periphery region becomes highly concentrated with the procession of the evaporation and this high density region is regarded as a circular ring of a capillary, and in which the cross-links were reconstructed to minimize the total free energy. The molecular may move easily rather than those of chemical gels, because the interaction among the MC gel molecules is weak force by van der Waals interaction and hydrogen bond.

Paracrystals were generated from nuclei while the high density MC gel turned to solidified thin film in the circular ring of a capillary during the drying process. We have already concluded in the previous paper ${ }^{7}$ that the cross-links of the MC gel compose fringed-micellar small crystallites, so that the cross-links might be the nuclei to generate the crystal domains.

Polymer chains of the MC gel in the circular ring of a capillary are considered to be oriented along the periphery of the ring to reduce the interaction energy among polymer chains and between polymer chains and glass substrate because of the weak interaction among the molecules in the physical gel. Therefore, the concentric circles of polymer chains may be generated.

Some studies have been reported about formation pattern generated by evaporation of solution, for example, Ozawa et al. reported about a dried droplet of silica solutions in which liquid becomes a gel in the middle of dried process ${ }^{12}$ and Okubo reported about the drying pattern formation of colloidal suspensions. ${ }^{13}$ However, their results are different from ours. Hence, we conclude that it is necessary to keep gel state all 


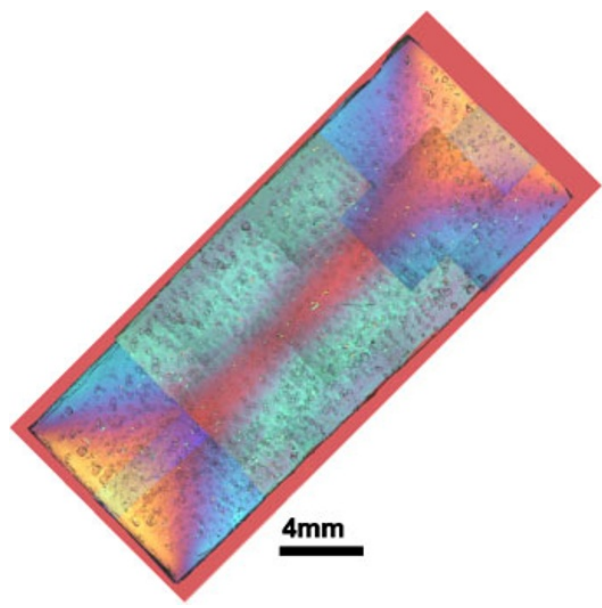

Figure 9. Image of dried film of $\mathrm{MC}$ gel (MC-4, $\left.C_{0}=3 \mathrm{wt} \%, T_{0}=65^{\circ} \mathrm{C}\right)$ with rectangular substrate observed by $\mathrm{POM}$ inserted sensitive color plate.

the time during drying process to obtain the self-assembled orientation of polymer chains as shown in our results. Moreover, we consider the interaction energy among MC chains is very adequate to make the self-assembled orientations, because the self-assembled films in our results are constructed by physical gel in which the molecular interaction is weaker than chemical gels but stronger than colloidal suspensions.

\section{Effect of the Shape of the Droplet}

In the case of circular droplet, the dried region appeared at the periphery and the polymer chains of MC gel were arranged parallel to the periphery to form concentric circles.

In order to investigate the effect of the shape of the periphery, a rectangular substrate was covered by the MC gel of $C_{0}=3 \mathrm{wt} \%$ of MC-4 solution and the drying temperature at $T_{0}=65^{\circ} \mathrm{C}$ was observed by POM. The result is shown in Figure 9. The dried region also appeared at the periphery and proceeds to the inside of the sample. As is shown in the figure, a negative spherulite-like pattern appeared in the whole area of dried rectangular film. The color patterns indicate that the polymer chains arranged on a parallel to the substrate edge. Therefore, the result suggests that the boundary of the sample plays an important role on the arrangement of the polymer chains of MC gels. In other words, we may control the orientation of the polymer chains by use of substrates having various shapes of the periphery.

\section{CONCLUSION}

Droplets of physical gel of MC on glass plates were dried up to form solidified thin films. The drying process was observed by POM and WAXS observation.

To obtain the characteristic self-assembled orientation of the MC polymer chains, it is important to select an adequate condition to keep in gel state during the drying process. Our observation made clear that differing from the sol originated samples, the POM patterns from our gel originated solidified thin films were very similar to those of a single negative spherulite. This suggests that polymer chains in the gel rearranged nearly on concentric circles around the center of the sample.

The polymer chains in the physical gel are supposed to move more easily than those in chemical gel in the concentrated area around the periphery of the droplet because the interaction among the molecules is weak. The orientation of the polymer chains was in the solidified thin film plane and was in parallel to the periphery of the film. The generation of this orientation may be caused to reduce the interaction energy among the polymer chains and between the polymer chains and the glass substrate. This self-assembling effect suggests that the drying process observed by us is peculiar to the process in the physical gel.

Our results suggest that when some particles having a function are dispersed in drying MC solution in gel state, the particles may be arranged together with the MC molecules which play a role of template and we can expect to obtain a new functional thin film such as a uniformly adsorbing hydrophobic or hydrophilic molecules by using the features of structural anisotropy of MC molecules for having hydrophilic and hydrophobic part in the cell unit.

Acknowledgment. The authors thank Prof. Takao Koda for helpful comments and Prof. Akira Kinbara for enlightening discussions in our manuscript.

Received: August 6, 2007

Accepted: November 6, 2007

Published: December 26, 2007

\section{REFERENCES}

1. T. Tanaka, S. Sun, Y. Hirokawa, S. Katayama, J. Kucera, Y. Hirose, and T. Amiya, Nature, 325, 796 (1987).

2. Y. Li and T. Tanaka, J. Chem. Phys., 92, 1365 (1990).

3. K. Hara, OYO BUTURI, 68, 290 (1999).

4. A. Suzuki and T. Hara, J. Chem. Phys., 114, 5012 (2001).

5. T. Yamaue and M. Doi, Phys. Rev. E, 69, 041402 (2004).

6. M. Takahashi, M. Shimazaki, and J. Yamamoto, J. Polym. Sci., Part B: Polym. Phys., 39, 91 (2001).

7. M. Takahashi and M. Shimazaki, J. Polym. Sci., Part B: Polym. Phys., 39, 943 (2001).

8. C. Yamane, T. Aoyagi, M. Ago, K. Sato, K. Okajima, and T. Takahashi, Polym. J., 38, 819 (2006).

9. G. Strobl, "The Physics of Polymers: Concepts for Understanding Their Structures and Behavior," Springer-Verlag, Berlin Heidelberg, 1996.

10. T. Kato, M. Yokoyama, and A. Takahashi, Colloid Polym. Sci., 256, 15 (1978).

11. P. Zugenmaier and A. Kuppel, Colloid Polym. Sci., 264, 231 (1986).

12. K. Ozawa, E. Nishitani, and M. Doi, Jpn. J. Appl. Phys., 44, 4229 (2005).

13. T. Okubo, Colloid Polym. Sci., 284, 1395 (2006). 